\title{
Semiclassical approach for all-atom nonadabatic simulations of excitation energy transfer processes in photosynthetic complexes
}

\author{
Hyun Woo Kim \\ Department of Chemistry \\ Pohang University of Science and Technology (POSTECH) \\ Pohang, 790-784, Korea \\ E-mail: highkhw@postech.ac.kr \\ Young Min Rhee ${ }^{1}$ \\ Center for Self-assembly and Complexity \\ Institute for Basic Science (IBS) \\ Pohang, 790-784, Korea \\ E-mail: ymrhee@ibs.re.kr
}

Recent experimental and theoretical studies have revealed that the excitation energy transfer (EET) can exhibit coherent characteristics in various photosynthetic systems. Applying an allatom nonadiabatic simulation technique will be extremely advantageous for elucidating the details of the involved processes, as it can offer microscopic views on protein-chromophore interactions. Here, we present how a semiclassical approach based on the Poisson bracket mapping equation formalism can be applied to the EET dynamics in the Fenna-Matthews-Olson complex. We show that the chromophore coupling maintained by the protein scaffold is the key factor for governing the coherence. The electrostatic modulations by the protein and the related site energy dispersion have only a limited effect. The role of thermal fluctuations in the chromophore couplings is additionally inspected in view of EET pathway diversity.

2013 International Workshop on Computational Science and Engineering (IWCSE 2013)

October 14-17, 2013

Taipei, Taiwan

${ }^{1}$ Speaker 


\section{Introduction}

Long-lived electronic coherences were observed for a few hundred femtoseconds in photosynthetic complexes even at the room temperature. ${ }^{1}$ These observations have gained much attention because they show that the quantum effect can be maintained in complex biological systems at physiological temperatures. This has also led to the development of many simulation methods mainly for revealing the role of coherences and their interplays with the protein environment. ${ }^{2,3}$ However, most theories are based on abstract bath models. Still, the role of protein is not explained unambiguously in atomic resolutions although it gives fundamental understanding on the coherent dynamics. To gain information at the atomic resolution, we summarize and review our recently published paper $^{4}$ on all-atom nonadiabatic simulations for the photosynthetic Fenna-Matthews-Olson (FMO) complex.

\section{Simulation method}

To simulate the nonadiabatic dynamics, our group extends an existing theory, Poisson bracket mapping equation ${ }^{5}$ (PBME) toward all-atom simulations. It may constitute a new class of theories in the photosynthetic research community as distinguished through three different classes of approaches (Figure 1). In many previous investigations, the bath information is considered with a spectral density that explains the environmental effect on the quantum system. Indeed, this model can give numerically exact results and attain important insights on the role of coherences. However, it cannot describe dynamics with atomic details as pictorially shown in Figure 1(a). To include the atomic information, energy transfer dynamics can be carried out after performing ground state molecular dynamics (MD) simulations with all-atom force fields (Figure 1(b)). Through atomic MD simulations, all-atom information is included in the dynamics. However, this class of models cannot explain the environmental relaxation properly because the system always feels the ground state bath. Therefore, we addressed a new class of all-atom models by simulating both energy transfers and the classical bath in the excited state as displayed in Figure 1(c).

(a)

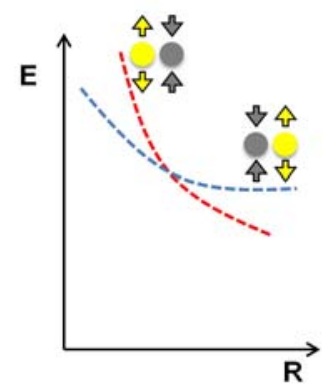

(b)

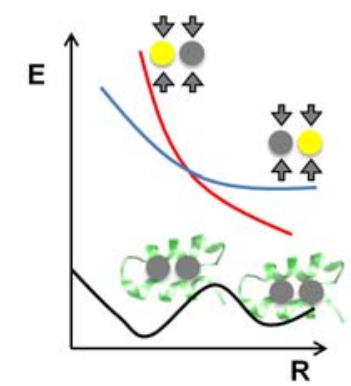

(c)

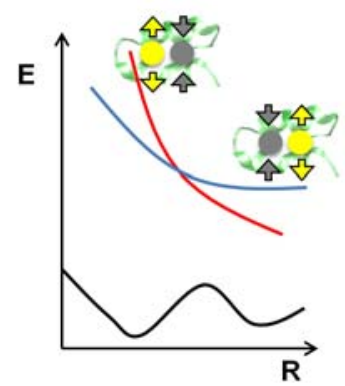

Figure 1. Comparison of nonadiabatic simulation methods. (a) Here, the bath information is simply incorporated in an abstract manner. In contrast, all atoms in the photosynthetic complexes can be treated in their (b) ground and (c) exited state configurations. 


\section{Results and Discussions}

With this model, we observed important characteristics of the coherent dynamics in the photosynthetic FMO complex. For example, we confirmed that the coherence can remain longer in a single complex than in the ensemble of complexes. Even with the exaggerated vibrational effect, the coherence could be observed in a single complex level. Moreover, we found that the protein structure is more important than the electrostatic effect in maintaining the coherence by directly comparing structural and electrostatic effects with all-atom simulations. For this, we simply modified the neighboring residues of sites 1 and 2 to alanines for structural mutation (Ala-mutant). To inspect the electrostatic effect from the protein, we ignored all atomic partial charges of the protein. As shown in Figure 2, coherent population transfer patterns are largely distorted in Ala-mutant while they are overlapping well with the wild-type populations in the electrostatic mutant. This implies that the coherence can be observed in the photosynthetic systems if the coupling between electronic states is not much varied.

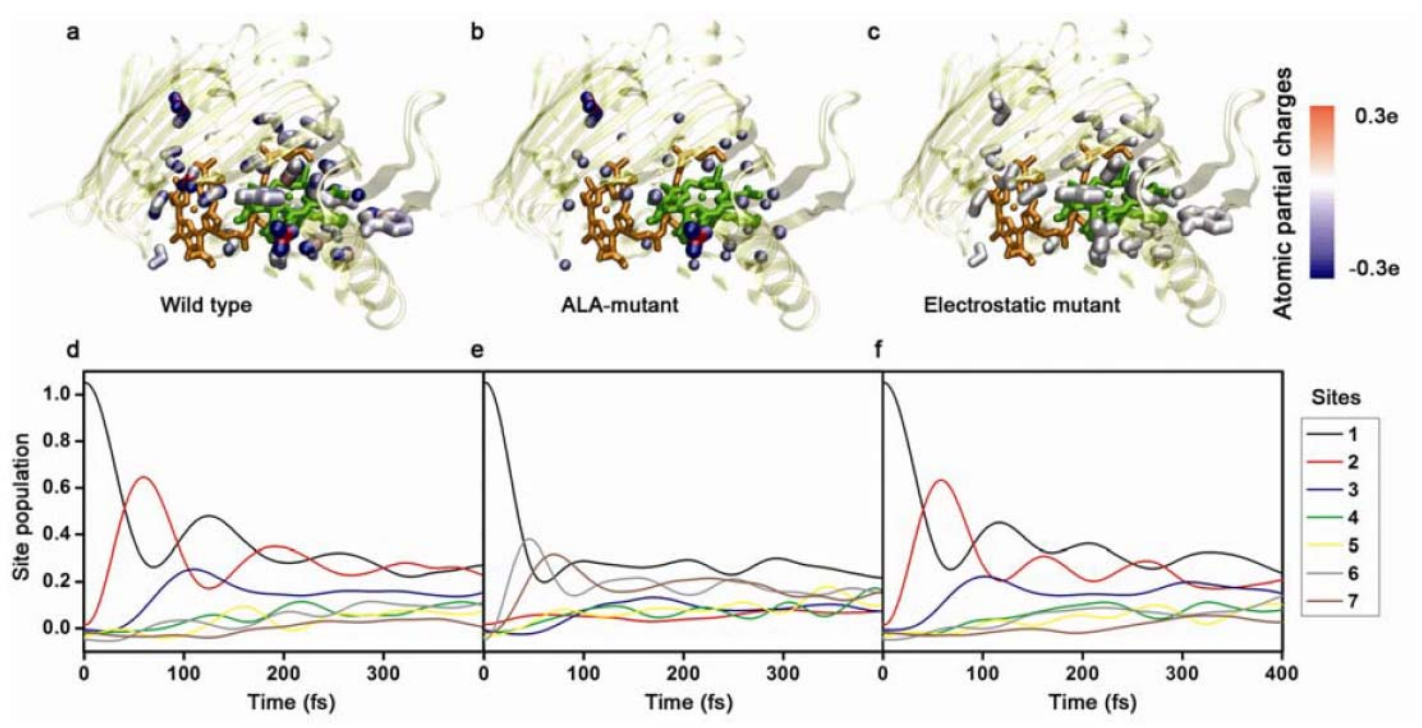

Figure 2. Structures of wild type, Ala-mutant, and electrostatic mutant with their population transfer patterns.

In addition, we investigated that the population dynamics initiated from a stationary state by preparing an eigenstate of the system Hamiltonian. In this case, the coherent oscillation is observed after $\sim 20$ fs. Based on this observation, we suggested that the coherence dynamics may be more general regardless of initial conditions. Finally, we showed the potential robustness of the photosynthesis, namely its resilience against external changes, by obtaining different population transfers in single complexes.

In summary, we simulated the coherent nonadiabatic dynamics in the photosynthetic FMO complex with atomic details and appropriate bath relaxations. We believe that this method can be applied to simulations of more complex systems in the future to better illustrate the photosynthetic energy transfer processes. 


\section{References}

[1] G. S. Engel, T. R. Calhoun, E. L. Read, T.-K. Ahn, T. Mančal, Y.-C. Cheng, R. E. Blankenship, and G. R. Fleming, Nature 446, 782 (2007).

[2] A. Ishizaki, T. R. Calhoun, G. S. Schlau-Cohen, and G. R. Fleming, Phys. Chem. Chem. Phys. 12, 7319 (2010).

[3] L. A. Pachón and P. Brumer, Phys. Chem. Chem. Phys. 14, 10094 (2012).

[4] H. W. Kim, A. Kelly, J. W. Park, and Y. M. Rhee, J. Am. Chem. Soc. 134, 11640 (2012).

[5] H. Kim, A. Nassimi, and R. Kapral, J. Chem. Phys. 129, 084102 (2008). 